\title{
Experimental Investigation on the Plasma Torch Used for Scramjet Ignition Enhancement
}

\author{
Wenli Zhong, Wenxiong Xi, Liwei Duan, Qingyao Xu, Qian Li \\ Academy of Equipment, Beijing, China \\ Email: zhongwenli1015@gmail.com
}

Received 20 April 2015; accepted 19 August 2015; published 26 August 2015

\begin{abstract}
A high frequency arc discharge plasma torch was specially designed for ignition enhancement in scramjet combustor. At first, the process of plasma injection into quiescent air was investigated experimentally through CCD camera and schlieren technology. Then, the energy property characterization of active particle distribution was measured by emission spectrometry. Several kinds of working gas under different injection pressures were compared. Finally, the typical supersonic flow-field structure with plasma cross-injection was obtained. The results show that plasma jet energy is concentrated near the jet axis, which has the maximum attenuation in the downstream as far as $\mathbf{2 ~ c m}$ from the outlet. The working gas and injection pressure have great effect on emission spectrometry and the process of jet expansion. The case with $\mathrm{N}_{2}$ under higher injection pressure shows better performance of energy exchanging process when comparing with air and argon. From the emission spectroscopy, we can see that plasma from nitrogen consists of nitrogen and oxygen atom mainly, whose intensity decreases with increasing distance from the nozzle, while it increases with the increase of pressure. When plasma was vertically injected into supersonic flow-field, bow shock wave and mixing layer structure were formed with thicken mixing layer, which helps enhance the mixing process between active particle and incoming air.
\end{abstract}

\section{Keywords}

Plasma Torch, Supersonic Flow, Schlieren Technology, Emission Spectroscopy, Shock Wave

\section{Introduction}

In recent years, with the development of aerospace technology, hypersonic engine technology has become a hot topic around the world [1]. Hypersonic engine has the ability to provide hypersonic flight for aircraft, but it brings another problem: supersonic combustion. In the process of supersonic combustion, gas flow has a high velocity and stays only a few milliseconds in the combustion chamber, which makes hydrocarbon fuel cannot be completely burned [2]. Plasma assisted ignition and combustion (PAIC) has drawn considerable attention as a potential way to improve supersonic combustion efficiency and propulsion systems [3]. Plasma is a quasineutral system, consisting of a large number of interacting particles, positive and negative particles, and a varie- 
ty of free radical reactive groups. The principle of plasma ignition and combustion is based on the fact that the core temperature of plasma jet can reach as high as $5000 \mathrm{~K}$, and non-equilibrium plasma polymerization release large amounts of heat. Therefore, the plasma ignition and combustion has the properties of high energy and strong penetration ability, which can help ignite high-speed fuel flow under harsh working conditions. There are many researches indicating that plasma ignition and combustion has many advantages such as: rapid heating flow, generation of active particles, achievement of large area ignition, shorten the ignition delay time, increasement of the rate of flame propagation, improvement of flame stability, broaden flammable limits, which makes it becomes the focus of extensive research countries [4]-[10]. The ways of plasma generation include: microwave discharges, pulsed nanosecond sparks [11], pulsed coronas, pulsed nanosecond dielectric barrier discharges [12] and volume nanosecond discharges [13] etc. The research area on the plasma ignition and combustion contains two aspects: numerical simulation and experimental verification, which shows that the active particles in the discharge gas products have big influence on the ignition and flame propagation property. In-depth study of plasma ignition characteristics and designing a safe and reliable plasma ignition system have great significance on the practical application of the plasma ignition system. Therefore, the goal of this paper is to study the ignition characteristics of the plasma ignition system experimentally, including analyzing plasma jet energy distribution property, measuring spectral characteristics of the igniter at the outlet by emission spectroscopy, and obtaining the typical supersonic flow-field structure with plasma cross-injection.

\section{Experimental System}

The plasma ignition experimental system contains several parts: high voltage power supply system, schlieren system, emission spectroscopy, high speed camera etc. The high voltage power supply system is shown in Figure 1, the operating frequency is $20 \mathrm{kHz}$, the output voltage is between $0-30 \mathrm{kV}$, the output power is between 0.5 - $5 \mathrm{~kW}$. Figure 2 shows the photo of plasma jet obtained by the sonic nozzle configuration.

As a non-intrusive flow visualization technique, schlieren technique can clearly obtain the shock wave structure of the flow field. In our experiment, we use high time and spatial resolution, as well as high sensitivity schlieren system to display the wave structure of the flow field structure. The schlieren system is shown in Figure 3. It mainly contains three parts: 1) pulsed xenon flash lamp source. 2) schlieren lens group with $150 \mathrm{~mm}$ in diameter, $1.5 \mathrm{~m}$ in focal length. 3) knife and imaging system.

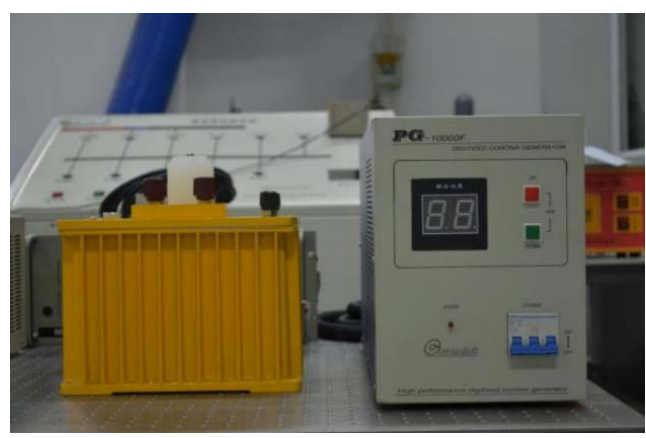

Figure 1. Photo of the high voltage power supply system.

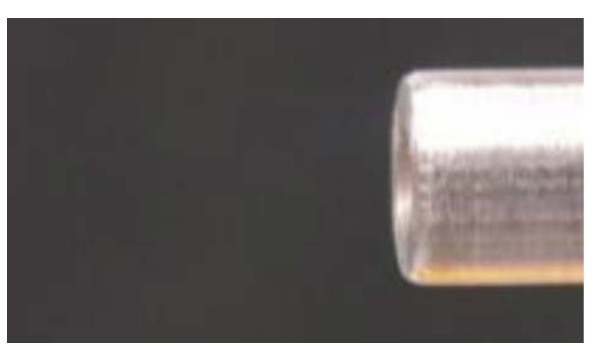

(a)

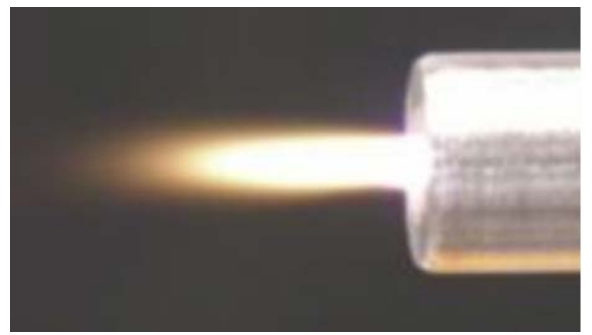

(b)

Figure 2. Photo of the obtained plasma jet by the power system. (a) Undischarged; (b) Discharged. 


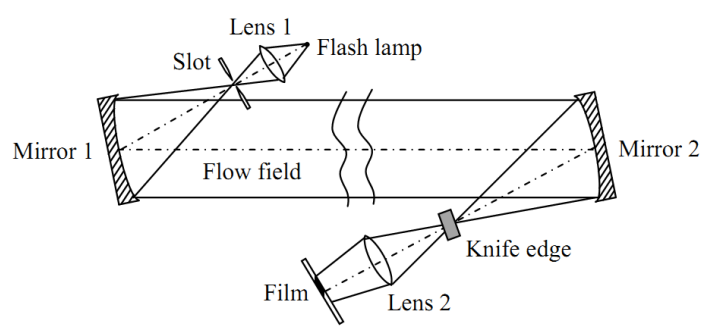

Figure 3. Schematic of schlieren experiment system.

The high-speed color camera we used in the experiment is Phantom ${ }^{\circledR}$ V711camera (Figure 4) produced by Vision Research Company of America, with field of view as $1000 \times 1000$ pixels, frame rate as high as 10,000 fps.

The plasma discharge parameters experimental device is shown in Figure 5, the angle between spectrometer fiber port and plasma jet is $90^{\circ}$, plasma refrigerants can be air, nitrogen or argon, the pressure changes between 4 and $6 \mathrm{~atm}$, the distance changes between 0 and $25 \mathrm{~mm}$, and we collected the spectrum signals by emission spectroscopy.

The emission spectroscopy we used in the experiment is shown in Figure 6, with type name as HR4000CGUV-NIR. Its bandwidth is $200-1100 \mathrm{~nm}$, with the entrance slit as $5 \mu \mathrm{m}$, and the filter to eliminate high order diffraction. The optical resolution is $0.7 \mathrm{~nm}$ FWHM, and the fiber diameter is $400 \mu \mathrm{m}$. We collected the emission spectrum between $200-1100 \mathrm{~nm}$.

\section{Results and Discussion}

In the process of plasma assisted ignition and combustion, plasma jet provide high temperature working fluid and active groups to the combustion chamber. The hot plasma jet energy contains three parts: jet momentum, jet enthalpy and active particles. Under different injection pressure and medium, the distribution of plasma jet form and active articles display different characteristics, which have great influence on the initial fire nuclear formation process. Therefore, we used the method of high speed camera and schlieren technology to get information on the energy distribution characteristics of the plasma jet, after that we had an idea on the composition and electron temperature of plasma jet by emission spectroscopy.

In the experiment, we selected three kinds of gas source to obtain plasma: air, nitrogen, argon. The relative property of these three kinds of gas is shown in Table 1. In the experiment, the injection pressure changed between 3 - 6 atm to study the difference on the property of plasma jet.

\subsection{Energy Distribution Characteristics of Plasma Torch}

When the working gas is nitrogen, under different injection pressures, the plasma torch configuration was recorded by high speed camera. When the exposure time was $100 \mu \mathrm{s}$, the photos were shown in Figure 7. As shown in Figure 7, plasma jet energy is concentrated near the jet axis, and the power decreases by exchanging the energy with the surrounding air. With the increasing injection pressure, the velocity of airflow around the jet exit increases, the output energy increases near the outlet. There is small difference in the axial energy attenuation characteristics: the energy has the maximum attenuation in the downstream as far as $2 \mathrm{~cm}$ from the outlet. In the radial direction, there is some difference in the energy attenuation property, the reason lies in two aspects: one is radial energy attenuation is determined by the shear properties between the plasma jet and the surrounding air, another is that with different injection pressure, arc property is different, energy transfer efficiency is different, leading to different airflow velocity.

When the working gas changed to air, with the adding $\mathrm{OH}$ filter, we had the photos shown in Figure 8(a). From Figure 8(a), we can see that there are $\mathrm{OH}$ groups in the plasma jet. $\mathrm{OH}$ group has ellipsoidal distribution in the downstream as far as $2 \mathrm{~cm}$ from the outlet. In the circumstance of $\mathrm{N}_{2}$, by adding NO filter, we could have the photo shown in Figure 8(b). It is concentrated in the downstream as far as $1 \mathrm{~cm}$ from the outlet. From $1 \mathrm{~cm}$ to $2 \mathrm{~cm}$, the intensity of NO unit decreases quickly, when the distance increases to $2 \mathrm{~cm}$, it almost disappears.

The degree of concentration of the jet momentum can display intuitively by the angle from the jet expansion. The big angle implies that energy exchanges strongly with the surrounding air, and energy distribution has large scope, but the jet penetration depth is limited in the supersonic flow. Figure $\mathbf{9}$ shows the schlieren images of the 


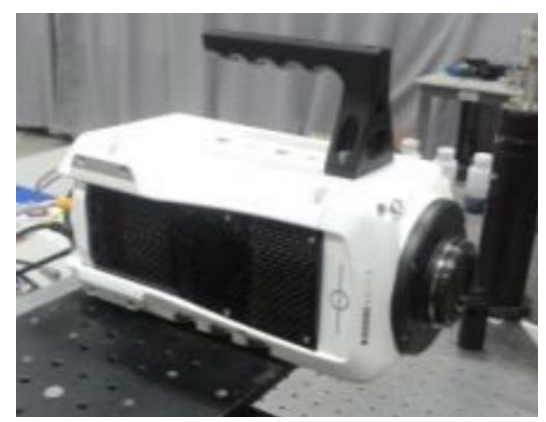

Figure 4. Photo of high-speed color camera.

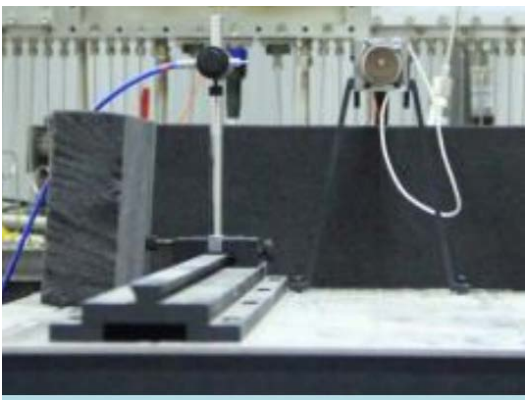

Figure 5. Schematic of plasma parameter experiment system.

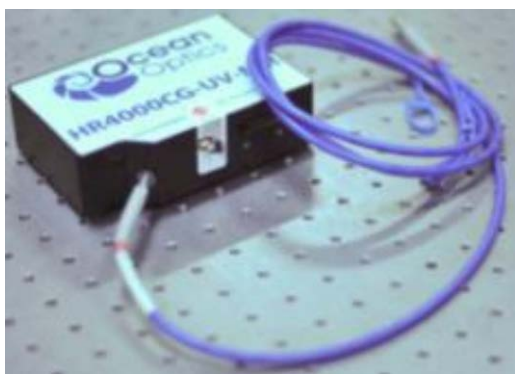

Figure 6. Photo of emission spectroscopy.

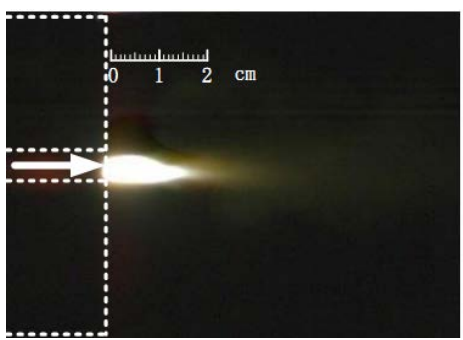

(a)

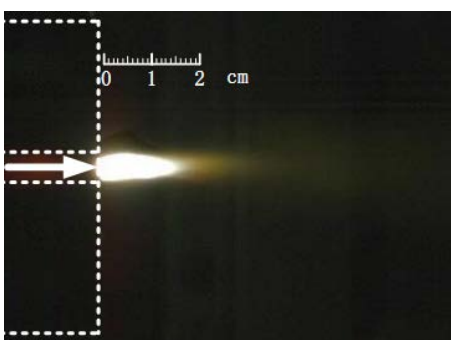

(b)

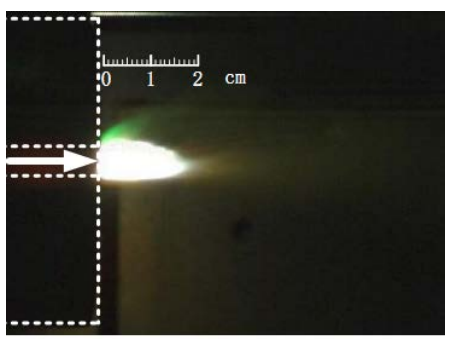

(c)

Figure 7. Photo of plasma jet from nitrogen in the quiescent air (a) 3 atm; (b) 4 atm; (c) 5 atm by high speed camera.

Table 1. The characteristics of working gas.

\begin{tabular}{cccc}
\hline \multirow{2}{*}{ Gas } & & Characteristics & \\
\cline { 2 - 4 } & Ignition Efficiency & Breakdown Characteristics & Damage to the electrode \\
\hline Air & high & hard & medium \\
Nitrogen & medium & medium & serious \\
Argon & low & easy & little \\
\hline
\end{tabular}




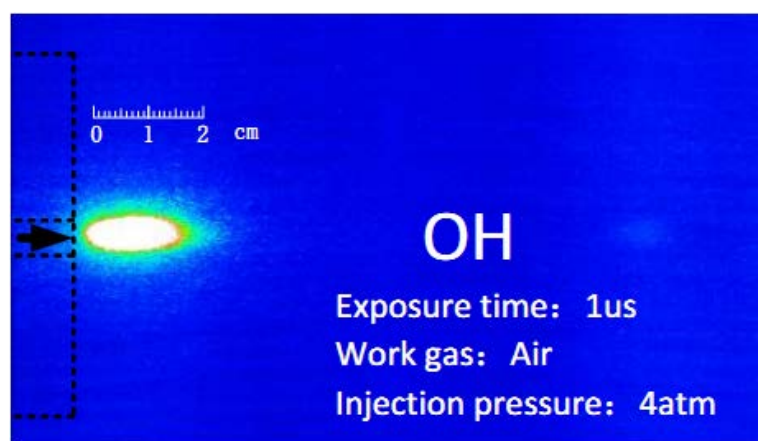

(a)

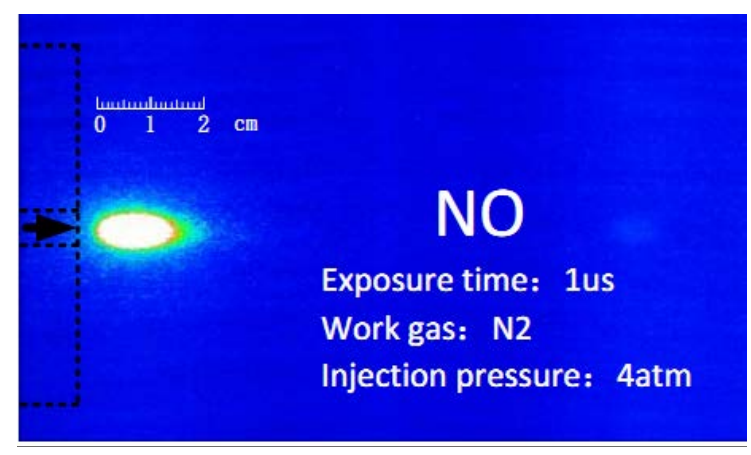

(b)

Figure 8. Photo of plasma jet with filters (a) $\mathrm{OH}$ filter (b) NO filter in the quiescent air by high speed camera.

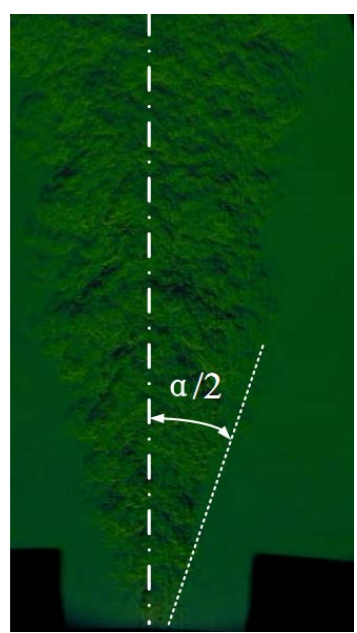

(a)

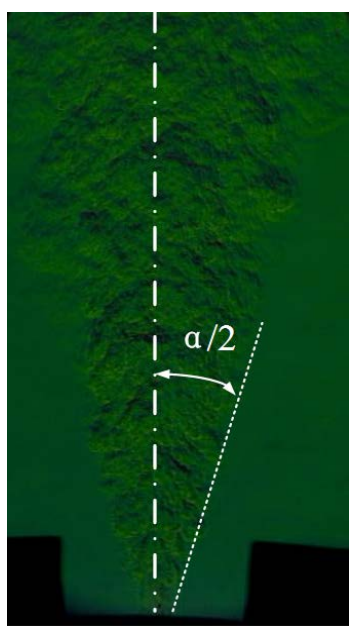

(b)

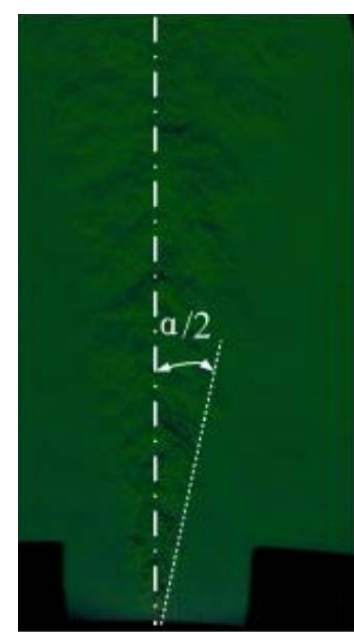

(c)

Figure 9. Schlieren images of the plasma jet from working gas (a) $\mathrm{N}_{2}, 5$ atm (b) Air, 5 atm (c) Ar, 5 atm in quiescent air.

plasma jet in different working gas with the injection pressure as $5 \mathrm{~atm}$. It is shown that with different working gas, the plasma jet expansion displays different angle size, with the sequence as: the plasma jet from nitrogen has the biggest angle, argon has the smallest angle, while air is in medium. The reason may lies in: there are many excitable active particles in nitrogen, such as molecules, atoms, ions (monovalent, divalent ions etc). Compared with air and argon, the concentration of the active particles belonging to nitrogen in the plasma is higher, which may lead to greater energy exchange process.

\subsection{Emission Spectroscopy Characteristics of Plasma Torch}

When the plasma working gas is air, the typical chromatogram is shown in Figure 10. Here, we present the emission spectrum when the injection pressure is $5 \mathrm{~atm}$, the distance between the plasma jet and the emission spectroscopy is $0 \mathrm{~mm}$. The peaks at $746.8 \mathrm{~nm}, 868.3 \mathrm{~nm}$ correspond to $\mathrm{N}$ atom, whereas the three peaks at 777.4 $\mathrm{nm}, 822.2 \mathrm{~nm}, 844.6 \mathrm{~nm}$ are attributed to $\mathrm{O}$ atom. In addition, the small peaks at $327.08 \mathrm{~nm}$ and $649.58 \mathrm{~nm}$ are the characteristic peaks of $\mathrm{O}^{+}$, whereas the peak at $517.95 \mathrm{~nm}$ belongs to $\mathrm{N}^{+}$.

The emission spectroscopy shows many oxygen atomic spectral lines, such as $777.4 \mathrm{~nm}, 822.2 \mathrm{~nm}, 844.6 \mathrm{~nm}$,

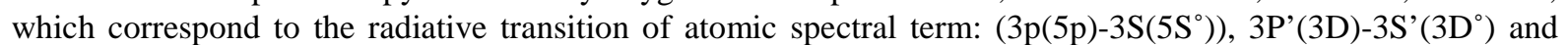
(3p(3p)-3S $\left(3 \mathrm{~S}^{\circ}\right)$ ). The excitation potential of these atomic transitions is $13.61 \mathrm{8eV}$, which implys that the plasma energy is greater than $13.618 \mathrm{eV}$.

In addition, it should be mentioned that the plasma jet under different injection pressure $(\mathrm{P}=4,5,6 \mathrm{~atm})$ display similar emission spectroscopy responses with the same number of bands attributable to the same groups. Only intensity of peaks differed. We performed the strength change curve of oxygen atom at $822.2 \mathrm{~nm}$ under different injection pressure and jet distance (Figure 11). As reported in Figure 11, spectra line intensity 


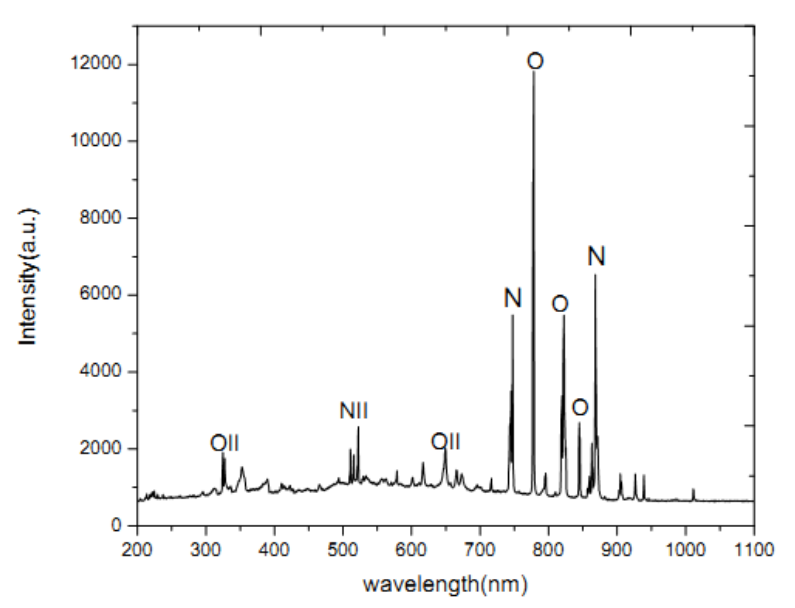

Figure 10. Emission spectra of plasma jet from air (distance between plasma jet and emission spectroscopy is $0 \mathrm{~mm}$ ).

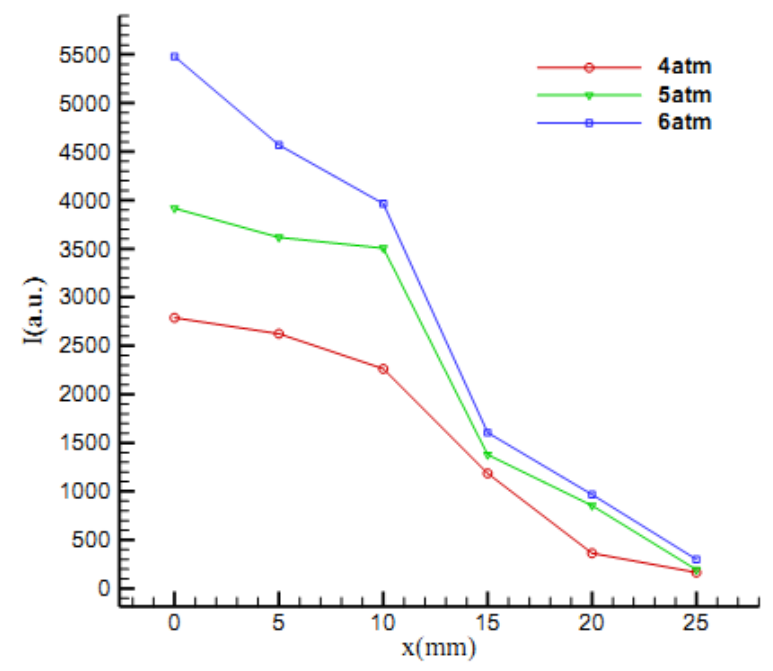

Figure 11. Evolution of oxygen atom $(822.2 \mathrm{~nm})$ intensity with the injection pressure and nozzle distance.

decreases with increasing distance from the nozzle, while it increases with the increase of pressure. When the optical fiber nears the plasma nozzle, $\mathrm{O}$ atom has the maximum intensity, which suggests that plasma has high energy; it has most excited oxygen atoms in the process of air breakdown. With the increase of distance, plasma energy decreases, leading to the decrease in the number of excited oxygen atoms. On the other hand, discharge pressure has big influence on the intensity of oxygen atom, especially in the nozzle position. The increasing voltage can significantly increase the plasma energy, to motivate more oxygen atom.

We have characterized the components of plasma with different working gas under the same experimental parameters. The emission spectrum recorded on plasmas from air and nitrogen with injection pressure as $5 \mathrm{~atm}$ and jet distance as $5 \mathrm{~mm}$ are presented in Figure 12. The plasmas both display the peaks belonging to nitrogen atom and oxygen atom with different peak intensity: the intensity of nitrogen atom (868.3 nm) in nitrogen is higher than in air, whereas the intensity of oxygen atom $(777.4 \mathrm{~nm})$ in nitrogen is lower than in air. The result shows that under nitrogen, plasma discharge mainly promotes the excited nitrogen atom process, the percentage composition of nitrogen atom is higher than oxygen atom.

When the plasma is argon, the typical emission spectra is shown in Figure 13. The curve in Figure 13 reveals that the plasma consists of a large number of Ar atoms in the excited state $(696 \mathrm{~nm}, 706 \mathrm{~nm}, 738 \mathrm{~nm}, 751 \mathrm{~nm}$, $763 \mathrm{~nm}, 772 \mathrm{~nm}, 794 \mathrm{~nm}, 801 \mathrm{~nm}, 811 \mathrm{~nm}, 826 \mathrm{~nm}, 842 \mathrm{~nm}$ ), without the formation of nitrogen and oxygen atom, which means that $\mathrm{Ar}$ atom is relatively easier to motivate than nitrogen and oxygen atoms. 


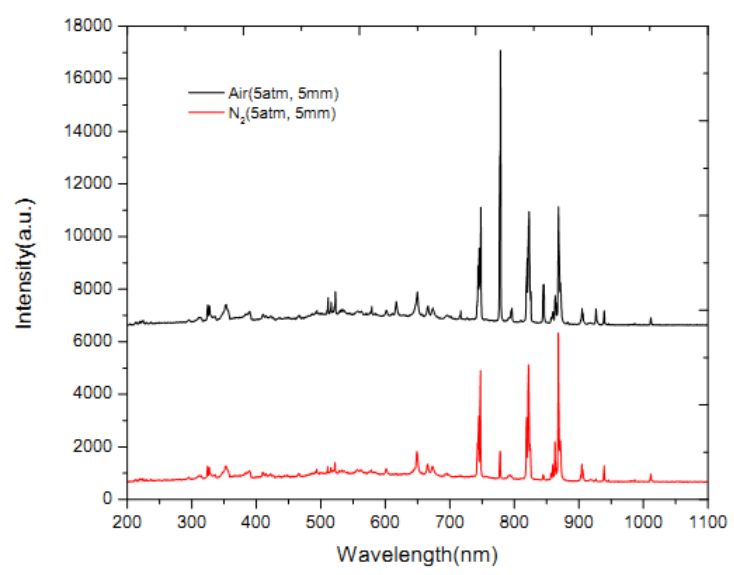

Figure 12. Emission spectroscopy of plasma under nitrogen and air (injection pressure: $5 \mathrm{~atm}$, distance: $5 \mathrm{~mm}$ ).

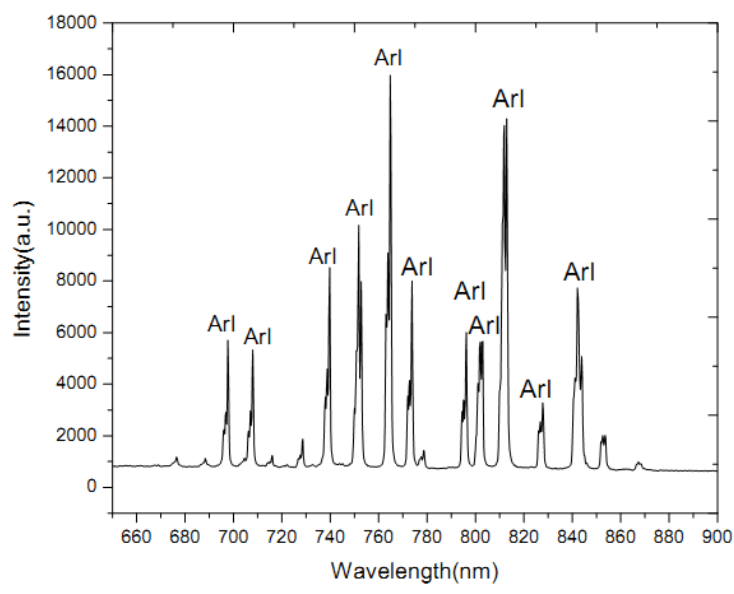

Figure 13. Emission spectroscopy of plasma under argon (injection pressure: $5 \mathrm{~atm}$, distance: $5 \mathrm{~mm}$ ).

\subsection{Evolution of Flow Structure with Plasma Torch Injection Vertically}

In order to get more information on the effect of plasma torch on the supersonic flow structure, the characteristic of density gradient was captured by schlieren system. Figure $\mathbf{1 4}$ gives the schlieren images in the circumstance of supersonic flow, and compared the image of flow structure with and without the addition of plasma torch.

The typical cavity flame stabilization chamber was used as the flow field model in the structure of scramjet combustion. The mainstream flow March number was set as 2, with the whole temperature as $300 \mathrm{~K}$, and the whole pressure as $5.6 \mathrm{~atm}$. It is shown in Figure 14 that because of the interaction between the plasma jet and supersonic flow, there is formation of bow shock wave and mixing layer structure to achieve the energy mixing process with the mainstream flow. The flow channel is filled with crossed reflex structure induced by shock wave. Plasma jet wake overlap together with the cavity shear layer in the downstream development process, increasing the thickness of shear layer. This process contributes to the inhalation of the active substances in plasma to the low speed concave cavity volume, which is the critical position to achieve flame stability. Through the experiments it is found that with the increase of injection pressure of plasma jet, the induced and reflected shock wave have greater strength, the energy mixing and exchanging process of the mainstream and plasma becomes more intense.

With the evolution of time, the plasma torch shape changed as illustrated in Figure 15. Because of the function of flow, the morphology of plasma torch has some changes: lump zone of energy concentration is pulled into strips, while the concentration of energy in the local position is still maintained very well, which can help provide sufficient ignition energy to cold fuel in the ignition process. 


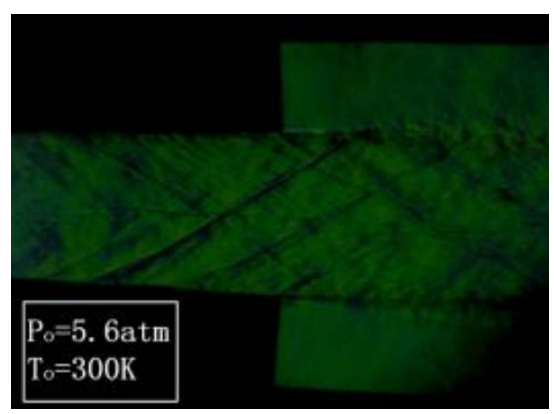

(a)

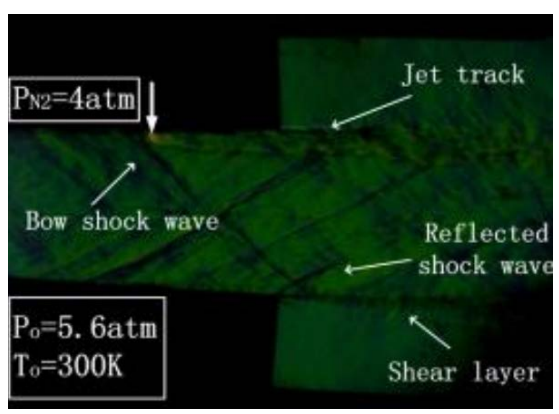

(b)

Figure 14. Schlieren images of supersonic flow structure: (a) without plasma; (b) with the addition of plasma torch vertically.

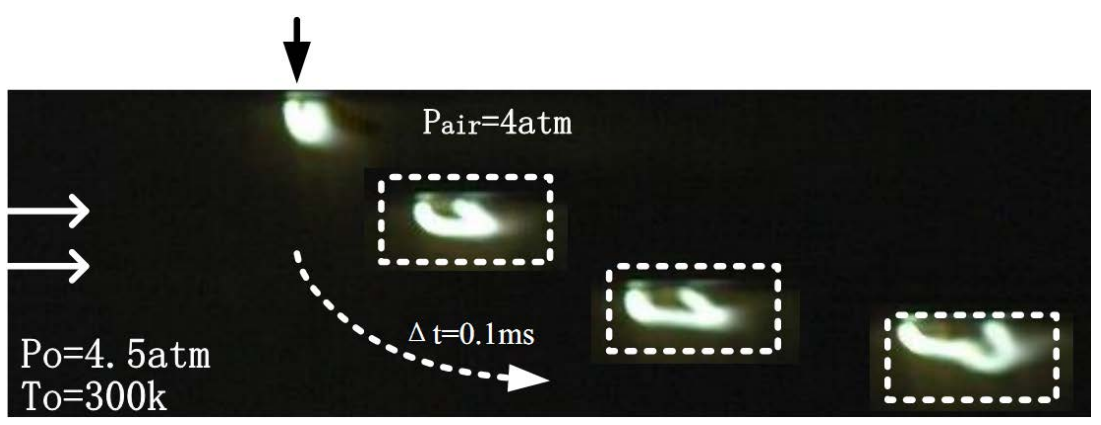

Figure 15. The shape evolution characteristics of plasma jet under the impact of transverse supersonic flow.

\section{Conclusions}

Through the experimental study of jet and discharge characteristics of plasma at the outlet of ignition machine by CCD camera and schlieren technology with different discharge gas and injection pressure, and according to the spectral characteristics of the plasma at the outlet of the ignition, we can have the conclusion as follows:

(1) Plasma jet energy is concentrated near the jet axis, and it has the maximum attenuation in the downstream as far as $2 \mathrm{~cm}$ from the outlet. When $\mathrm{N}_{2}$ is discharged, with the increase of injection pressure from 3 to $5 \mathrm{~atm}$, the velocity of airflow around the jet exit increases, and the output energy near the outlet increases.

(2) Plasma with different working gas has different jet momentum. Compared with air and argon, plasma from nitrogen has bigger angle from the jet expansion, which implies energy exchanges stronger with the surrounding air, and energy distribution has larger scope.

(3) Plasma from nitrogen and air consists of nitrogen atom and oxygen atom mainly, whose intensity decreases with increasing distance from the nozzle, while it increases with the increase of pressure. Plasma from argon consists of a large number of Ar atoms, without the formation of oxygen and nitrogen atoms.

(4) Because of the interaction between the plasma jet and supersonic flow, there is formation of bow shock wave and mixing layer structure to achieve the energy mixing process with the mainstream flow. Plasma jet wake overlap together with the cavity shear layer in the downstream development process, increasing the thickness of shear layer.

\section{References}

[1] Fry, R.S. (2004) A Century of Ramjet Propulsion Technology Evolution. Journal of Propulsion and Power, 20. http://dx.doi.org/10.2514/1.9178

[2] Law, C.K. (2006) Combustion Physics. Cambridge University Press. http://dx.doi.org/10.1017/CBO9780511754517

[3] Starikovskaia, S.M. (2006) Plasma Assisted Ignition and Combustion. J. Phys. D: Appl. Phys, 39, R265-R299. http://dx.doi.org/10.1088/0022-3727/39/16/r01

[4] Starikovskaya, S.M., Kukaev, E.N. and Kuksin, A.Y. (2004) Analysis of the Spatial Uniformity of the Combustion of a 
Gaseous Mixture Initiated by a Nanosecond Discharge. Combustion and Flame, 139, 177-187. http://dx.doi.org/10.1016/j.combustflame.2004.07.005

[5] Bozhenkov, S.A., Starikovskays, S.M. and Starikovskii, A.Y. (2003) Nanosecond Gas Discharge Ignition of $\mathrm{H}_{2}$ and $\mathrm{CH}_{4}$ Containing Mixtures. Combustion and Flame, 133, 133-146. http://dx.doi.org/10.1016/S0010-2180(02)00564-3

[6] Starikovskii, A.Y. (2005) Plasma Supported Combution. Proceeding of the Combustion Institute, 30, 2405-2417. http://dx.doi.org/10.1016/j.proci.2004.08.272

[7] Aleksandrov, N.L., Kindysheva, S.V. and Kosarev, I.N. (2009) Mechanism of Ignition by Non-Equilibrium Plasma. Combustion and Flame, 32, 205-212. http://dx.doi.org/10.1016/j.proci.2008.06.124

[8] Kosarev, I.N., Aleksandrov, N.L. and Kindysheva, S.V. (2008) Kinetics of Ignition of Saturated Hydrocarbons by Nonequilibrium Plasma: $\mathrm{CH}_{4}$ Containing Mixtures. Combustion and Flame, 154, 569-586. http://dx.doi.org/10.1016/j.combustflame.2008.03.007

[9] Kosarev, I.N., Aleksandrov, N.L. and Kindysheva, S.V. (2009) Kinetics of Ignition of Saturated Hydrocarbons by Nonequilibrium Plasma: $\mathrm{C}_{2} \mathrm{H}_{6}$ to $\mathrm{C}_{5} \mathrm{H}_{12}$ Containing Mixtures. Combustion and Flame, 156, 221-233. http://dx.doi.org/10.1016/j.combustflame.2008.07.013

[10] Andrey Starikovskiy, N.A. (2013) Plasma-Assisted Ignition and Combustion. Progress in Energy and Combustion Science, 39, 61-110. http://dx.doi.org/10.1016/j.pecs.2012.05.003

[11] Pancheshnyi, S., Lacoste, D.A., Bourdon, A. and Laux, C.O. (2005) Ignition of Propane-Air Mixtures by Repetitively Pulsednanosecond Gas Discharges. Proc. 17th Int. Symp. on Plasma Chemistry, Toronto, 7-12 August 2005.

[12] Takita, K. (2002) Ignition and Flame-Holding by Oxygen, Nitrogen and Argon Plasma Torches in Supersonic Airflow. Combust. Flame, 128, 301-313. http://dx.doi.org/10.1016/S0010-2180(01)00354-6

[13] Bozhenkov, S.A., Starikovskaia, S.M. and Starikovskii, A.Yu. (2003) Nanosecond Gas Discharge Ignition of $\mathrm{H}_{2-}$ and $\mathrm{CH}_{4}$-Containing Mixtures. Combust. Flame, 133, 133-146. http://dx.doi.org/10.1016/S0010-2180(02)00564-3 